\title{
El lenguaje evaluativo como mecanismo argumentativo de las cuentas \#studygram en tiempos de pandemia del covid-19
}

\section{The evaluative language as an argumentative mechanism of the \#studygram accounts in the COVID-19 pandemic timesa}

\author{
Natalia Wiśniewska* \\ *Universidade de Bielsko-Biała, Bielsko-Biała/Polonia \\ nwisniewska@ath.bielsko.pl \\ http:/ / orcid.org/0000-0001-7860-7062
}

\begin{abstract}
RESUMEN: En este artículo, de carácter descriptivo y cualitativo, analizaremos brevemente el lenguaje evaluativo utilizado por los estudiantes en las cuentas de Instagram de tipo \#studygram. La investigación se basa en la teoría de la argumentación en la lengua enriquecida por el punto de vista axiológico. Asimismo, el análisis se llevará a cabo en el contexto de la pandemia del covid-19 que ha marcado el discurso actual de varios ámbitos. El presente artículo sirve, por un lado, para presentar qué tipo del léxico evaluativo utilizan los estudiantes en una de las redes sociales más exitosas y, por el otro, para mostrar que el problema del covid-19 se puede investigar no solamente desde la perspectiva médica o de la enseñanza online, sino también tomando en consideración la actitud individual de los estudiantes.
\end{abstract}

PALABRAS CLAVE: lenguaje evaluativo; Covid-19; redes sociales; Instagram; \#studygram.

ABSTRACT: This paper, of descriptive and qualitative nature, briefly studies the evaluative language used by students in \#studygram-type Instagram accounts. The research is based on the basic concepts of the theory of argumentation in language endowed with the axiological point of view. Likewise, the analysis will be carried out in the context of the COVID-19 pandemic that has marked the current discourse in several areas. This article serves, on the one hand, to present what type of evaluative lexicon students use in one of the most successful social networks and, on the other hand, to show that the problem of COVID-19 can be investigated not only from a medical or online teaching perspective, but also by taking into consideration the individual attitude of students.

KEYWORDS: evaluative language; COVID-19; social media; Instagram; \#studygram. 


\section{Introducción}

La situación actual que experimenta el mundo desde principios del año 2020 por la pandemia del covid-19 se relaciona estrechamente con todas las esferas de la vida humana. En consecuencia, surgen los trabajos que se ocupan del problema desde la perspectiva de la medicina o virología, que tratan de distintos aspectos del virus. (BASTARD et al., 2020; MEHTA et al., 2020; MORALES CHÁVEZ, 2020; NISHIURA et al., 2020; VILLASEÑOR LÓPEZ et al., 2021) Asimismo, los científicos y los profesores se preocupan por la calidad de la enseñanza online desde la escuela primaria hasta las universidades y, por lo tanto, empiezan a buscar nuevos modelos con base en las tecnologías de la información y comunicación (TIC) que sean eficientes y cumplan varios requisitos formales establecidos por la ley. (ALVES; LOPES; PRECIOSO, 2020; FERNÁNDEZ MARTÍNEZ et al., 2020; MORENO MARTÍNEZ; MORALES CEVALLOS, 2020) Si tomamos en consideración la economía tanto a nivel nacional como a nivel mundial, observamos comentarios sobre distintas áreas de comercio (CABRER BORRAS; RICO, 2020; KASHYAP; RAGHUVANSHI, 2020; TORRES; FERNÁNDEZ, 2020), pero también respecto al mercado laboral. (LLORENTE HERAS, 2020; MOEN; PEDTKE; FLOOD, 2020; MONTENOVO et al., 2020; SPURK; STRAUB, 2020)

En cuanto a las investigaciones del ámbito de la lingüística, los autores se interesan sobre todo por el discurso político (DÍEZ GUTIÉRREZ; GAJARDO ESPINOZA, 2020; LLANO GUIBARRA; AGUILA SÁNCHEZ, 2020; PÉREZ-CURIEL; VELASCO MOLPECERES, 2020), pero también hay estudios que tratan del discurso científico, como, por ejemplo, Clavijo Naula y Cabrera (2020) o Dantas y Deccache-Maia (2020). Asimismo, resalta la importancia del análisis del lenguaje periodístico, en muchos casos de las portadas y titulares. (ÁLVAREZ-ROSA; ROMERO LÓPEZ, 2020; ARGIÑANO; GOIKOETXEA BILBAO, 2020) Este tipo de discurso se estudia también desde el punto de vista de la cualidad de información, lo que pueden confirmar trabajos que analizan, en general, los bulos informativos, llamados fake news, tanto desde la perspectiva propiamente lingüística (DOMINGUES ALMEIDA; SANTOS, 2020; GUTIÉRREZ COBA; COBA GUTIÉRREZ; GÓMEZ DIAZ, 2020; PIRES FRANCO et al., 2020), como de las ciencias de comunicación (GARCÍA MARÍN, 2020; VILLA GRACIA; CERDÁN MARTÍNEZ, 2020). 
Hemos observado que, a la hora de conocer distintas problemáticas que han surgido últimamente respecto al covid-19, muchos de los trabajos, también los que hemos mencionado anteriormente, se centran sobre todo en unas cuestiones bastante generales y muy serias, lo que no es nada raro si tomamos en consideración la situación mundial sin precedentes. Asimismo, se ha notado que, aunque muchos trabajos traten de los estudiantes o de la enseñanza online, las investigaciones se ocupan mayoritariamente de los problemas de naturaleza social (VIVANCO SARAGURO, 2020), del ámbito de psicología o pedagogía y metodología de distintas materias. (ALVES; LOPES; PRECIOSO, 2020; FERNÁNDEZ MARTÍNEZ et al., 2020; GARCÍA MEJÍA; GARCÍA VERA, 2020) No obstante, creemos que la situación de los estudiantes durante la pandemia no debería verse solamente desde la perspectiva de algún sistema educativo mejor o peor preparado para la situación, sino que es necesario girarse también hacia los mismos estudiantes que, como muchos otros grupos sociales, han tenido y tienen que ajustar buena parte de su vida a la nueva realidad. Por consiguiente, en el presente trabajo pretendemos poner énfasis no en la enseñanza durante la pandemia, sino en el lenguaje utilizado en esta época por los estudiantes que, sin duda alguna, son la parte fundamental del sistema educativo. Nos centramos en describir brevemente uno de los mecanismos argumentativos, el lenguaje evaluativo, que permite a los estudiantes expresar todo lo que consideran importante para ellos en esta nueva realidad. Sin embargo, nuestro estudio no se aleja de lo que hoy entendemos como algo imprescindible en la vida cotidiana, es decir, del mundo virtual. Como sabemos que la pandemia y el confinamiento han cambiado las redes sociales en una de las herramientas principales de la comunicación entre los seres humanos, tomamos como punto de partida Instagram, que durante los últimos años se ha convertido en una de las plataformas más utilizadas entre los jóvenes. Así nuestro objetivo es observar qué lenguaje utilizan los estudiantes en las cuentas de Instagram de tipo \#studygram.

\section{Los jóvenes en las redes sociales}

La omnipresencia de Internet y de las redes sociales en nuestra vida cotidiana se nota perfectamente entre las personas más jóvenes: los adolescentes y los estudiantes. Las últimas generaciones de los jóvenes, es decir, la Generación Y o Millennials (nacidos entre 1980-1999), la 
Generación Z (nacidos entre 2000-2010) y Generación Alpha/Glass (nacidos desde 2010), son las que ya están acostumbradas a la vida virtual, aunque cada una de ellas la percibe de otra manera. (GONZÁLEZ HERNANDO; VALDIVIESO LEÓN; VELAZCO GONZÁLEZ, 2020; ORTEGA; SOTO, 2016) No obstante, son las generaciones Y y Z que corresponden actualmente a la mayoría de los estudiantes universitarios o a las personas que siguen alguna formación, entre otras.

En lo que atañe a los Millenials, Bernal Galindo (2015) recopila que son las personas que han crecido junto al desarrollo de la tecnología, sienten fascinación hacia la red, están conectadas constantemente viviendo online y offline y saben perfectamente trabajar con varias pantallas a la vez. En el ámbito de educación, las personas de esta generación se caracterizan por estar expuestas a todo tipo de estimulación, lo que implica una atención limitada, aunque de varios asuntos a la vez, y un aprendizaje rápido si el tema les resulta interesante; además, son dependientes de la tecnología y dan como válida toda la información que les llega de Internet y de las redes sociales (BONGARRÁ, 2011).

La generación $Z$, en cambio, se compone de personas que nacieron y crecieron con los dispositivos móviles, cuando Internet y las redes sociales ya se habían fortalecido en la vida cotidiana. (GONZÁLEZ HERNANDO; VALDIVIESO LEÓN; VELAZCO GONZÁLEZ, 2020) Verdú (2015) destaca que son los jóvenes que ya "no se conforman con ser sujeto pasivo de marcas y publicaciones", sino que quieren producir sus propios contenidos, por ejemplo, en YouTube. (CONDE; FORTEZA MARTÍNEZ; ANDRADE MARTÍNEZ, 2020; VIZCAÍNO VERDÚ; CONTRERAS PULIDO, 2020) Si se trata de la educación, en general no creen en su forma tradicional y prefieren realizar el aprendizaje fuera del aula, aprenden en Internet usando YouTube o buscando información online. (VERDÚ, 2015)

De todo ello, en la respuesta a las necesidades de la gente joven cuyo aprendizaje se realiza, ya sea en el aula, ya sea a través de la educación informal, muy a menudo digitalizada, han surgido modelos que se sirven de varias plataformas propias de Internet, como los blogs (MOLINA AVENTOSA; VALENCIANO VALCÁRCEL; VALENCIA PERIS, 2015; MONTANER VILLALBA, 2019) o las redes sociales. (MEDINA, 2020; SÁNCHEZ PACHECO, 2020) Conviene resaltar, siguiendo a Liu (2010), que ninguno de estos medios sociales más utilizados por los jóvenes ha sido diseñado con fines de aprendizaje. En la mayoría de los casos se trata de 
fines recreativos como "juegos, comunicación y configuración de espacios en línea para la expresión de identidad personal”. (ARAB; DÍAZ, 2015, p. 8) No obstante, los profesores del siglo XXI se dan cuenta de la fuerza de la red y han empezado a aprovecharla en el campo de la educación (ESPINOZA; TINOCO IZQUIERDO; SÁNCHEZ BARRETO, 2017; SÁNCHEZ PACHECO, 2020).

\subsection{Las cuentas \#studygram en Instagram}

Si bien es cierto que los docentes se sirven de todo tipo de plataformas con fines de aprendizaje, no es menos obvio que también son los mismos estudiantes que comienzan a publicar informaciones de distinta índole. De ahí merece la pena mencionar los internautas que se mueven con éxito por la plataforma más joven, Instagram, que apareció en el año 2010 con el objetivo de ofrecer a los internautas posibilidades de contacto a través de las fotografías y se ha convertido en una de las redes sociales más utilizadas (FONT MARSAL, 2015; THE SOCIAL MEDIA FAMILY, 2020).

Su funcionamiento se basa sobre todo en la versión dedicada a teléfonos inteligentes o tabletas que se puede descargar gratis desde Google Play o App Store. (FONT MARSAL, 2015) Los usuarios crean allí sus nombres y pueden gozar de un "muro", o sea, un espacio donde se observan las fotografías compartidas por los internautas que "seguimos". Además, si empezamos a utilizar la aplicación, podemos compartir "historias" que, si no se guardan en "destacados", desaparecen después de 24 horas. Asimismo, en Instagram los internautas pueden mejorar sus fotografías a través de filtros digitales o vincular el perfil con otras redes sociales, como Facebook, Twitter, Tumblr o Flickr (BUCZEK, 2017; FONT MARSAL, 2015).

Como todas las redes sociales, también Instagram sirve para la comunicación: los usuarios pueden mandar mensajes instantáneos, dejar los comentarios debajo de cada una de las publicaciones o responder al contenido que aparece en "historias". Es más, se puede dejar un "like" a una publicación, enviarla a otros o facilitarla en su "historia". Otra característica de Instagram se relaciona con la posibilidad de ordenar publicaciones a base de hashtags o etiquetas, es decir, palabras o expresiones precedidas por una almohadilla o numeral (\#). (KOPEĆ, 2015) Así, como recopila Llorente (2019), los hashtags deberían describir bien el tema de una publicación, lo que ayuda a los usuarios a encontrar contenidos afines a sus intereses. De esta manera, la herramienta aumenta la posibilidad de encontrar el mensaje 
o la fotografía por parte de otros usuarios, de lo que se aprovechan tanto los usuarios privados como los influencers o las marcas de distintos productos (BUCZEK, 2017; LLORENTE, 2019).

Todo esto tiene relaciones con el mundo de los estudiantes, no porque sean personas que tienen cuentas privadas de Instagram, sino porque muchos han entrado en el mundo de las cuentas llamadas \#studygrams. ${ }^{1}$ Aunque es un fenómeno emergente que abarca muchas cuestiones, las personas que desarrollan cuenta de este tipo, es decir, \#studygramers, se pueden definir, según lo presentan Izquierdo Iranzo y Gallardo Echenique (2020, p. 120), como "personas que exponen sus apuntes, comparten experiencias de su vida estudiantil, ofrecen consejos sobre planificación y estudio, y, a veces, resuelven dudas".

No obstante, conviene destacar que, de acuerdo a Megía (2017) y Olmo (2017), no se trata solamente de compartir las experiencias de la vida estudiantil, sino de presumir de ser un empollón o una empollona. En muchos casos, como destaca Megía (2017), tal actividad estudiantil en la red puede influir de manera positiva en los resultados en los exámenes, en su motivación o disciplina para lograr los objetivos. Además, conviene destacar que la colectividad de \#studygramers se conforma de personas que no tienen problemas de compartir sus apuntes, dar trucos para aprender más rápido u ofrecer algunos productos gratis. (HARKER, 2019) De tal manera, observamos que, lo que para algunos podía ser un problema entre los niños o los estudiantes que en la vida real vivían con la opinión negativa de ser empollones, ahora se convierte en algo que vale la pena presentar a los demás.

Si tomamos en consideración el aspecto visual en el que se basa el funcionamiento de la plataforma, es imprescindible mencionar que las fotos que aparecen en este tipo de cuentas muestran todos los elementos de la vida estudiantil, aunque podemos notar que no existe un solo modelo de imágenes que se suelen colgar. Así, en los perfiles de tipo \#studygram sus seguidores pueden encontrar fotos de la caligrafía del usuario, su espacio de estudio, bolígrafos y rotuladores, cuadernos, libros y ordenadores, es decir, todo lo que se necesita para estudiar (MEGÍA, 2017; OLMO, 2017).

\footnotetext{
${ }^{1}$ Aunque Izquierdo Iranzo y Gallardo Echenique (2020) subrayan que existen también otras variantes, como \#studigram, \#estudygram o \#estudigram, nosotros hemos optado por la versión \#studygram que se emplea más entre los usuarios.
} 
Desde otro punto de vista, con todo lo positivo que contienen cuentas de este tipo, conviene subrayar que Instagram, igual a otras redes sociales, también puede crear distintas formas de vicio entre los usuarios. (MONTEIRO et al., 2020) Además, los profesores y los padres señalan que publicar en la red cómo se estudia no significa que los estudiantes lo hagan de verdad. (TIFFANY, 2017) Sin embargo, creemos que estas cuestiones deberían ser estudiadas más bien por las ciencias pedagógicas o sociales y, por tanto, nuestro trabajo se enfoca solamente en uno de los mecanismos lingüísticos utilizados por los \#studygramers.

Resumiendo, creemos que Instagram es un lugar de encuentros virtuales tanto de la gente famosa como de personas normales de varias generaciones. Así, a este último grupo pertenecen también los alumnos, los estudiantes universitarios y, en general, las personas que siguen formándose en algún ámbito. Sus cuentas llamadas \#studygram, aunque sean un fenómeno reciente, pueden formar bases para el estudio de corte lingüístico de varios niveles de la lengua. Además, en el contexto actual de la pandemia del covid-19, el uso de estas cuentas resulta ser aún más importante, ya que puede ofrecer otro modelo de aprendizaje en Internet (IZQUIERDO IRANZO; GALLARDO ECHENIQUE, 2020).

\section{Fundamentos teóricos}

La facilidad del uso de Internet y el acceso casi ilimitado a las redes sociales hacen que la esfera online de nuestra vida se convierta en la esfera pública que, según Habermas (2008), es un espacio donde cada uno puede participar en el discurso sociopolítico y confrontar sus opiniones. Para Navarro Colorado (2003), este espacio abierto, sin límites físicos, es propiamente persuasivo, ya que todas las páginas web y, en el contexto actual para nosotros también las redes sociales, tienen por objetivo alcanzar el interés de los internautas.

Desde el punto de vista de la lingüística, este carácter persuasivo se estudia también en los actos argumentativos desde hace años. (ANSCOMBRE; DUCROT, 1994; LO CASCIO, 1998; PERELMAN; OLBRECHTS-TYTECA, 1989; PLANTIN, 2005) Según Anscombre y Ducrot (1994), los autores de la teoría de la argumentación en la lengua, todos los textos tienen por objetivo convencer al otro haciendo uso del lenguaje verbal y no verbal. El acto de persuadir se lleva a cabo basándose 
en las nociones de fuerza y de orientación argumentativas marcadas en el discurso a través de los operadores que guían a los receptores del mensaje a la hora de sacar las conclusiones de los argumentos dados. Los operadores se comprenden como morfemas que, aplicados a un enunciado, pueden cambiar el potencial argumentativo de su contenido. (ESCANDELL VIDAL, 2007) Martín Zorraquino y Portolés (1999, p. 4139) añaden que los operadores "condicionan las posibilidades argumentativas del miembro del discurso en el que se incluyen, pero sin relacionarlo con otro miembro anterior".

No obstante, Fuentes Rodríguez y Alcaide Lara (2007) van más allá al subrayar que la fuerza y la orientación argumentativas no dependen solamente de los operadores típicamente argumentativos, tal y como lo definen Anscombre y Ducrot (1994), sino también de otros elementos lingüísticos: los conectores, la acumulatio, la repetición de expresiones y estructuras sintácticas, los elementos modales de reafirmación o el léxico en sí mismo.

De tal manera, por lo que se refiere al propio vocabulario que sirve para transmitir un mensaje dado, la cuestión de lenguaje evaluativo se inscribe dentro del funcionalismo, en lo cual se hace énfasis en el uso de la lengua y las funciones socioculturales que se estudian desde diferentes perspectivas. (CONRAD; BIBER, 2000; HUNSTON; SINCLAR, 2000; HUNSTON; THOMPSON, 2000; MARTIN; ROSE, 2008; MARTIN; WHITE, 2005) Pascual (2011, p. 1) recopila que, aunque los enfoques muestran diferencias, se refieren ampliamente a "la evaluación como la expresión de actitudes, juicios, posicionamientos o sentimientos de los escritores o hablantes tanto hacia las entidades o proposiciones a las que hacen referencia como hacia sus interlocutores". En general, se toma en consideración, por un lado, la orientación del elemento evaluado que puede ser negativa o positiva y, por el otro, los valores que pueden ser juzgados. Kerbrat-Orecchioni (1986, p. 93) destaca que un sujeto a la hora de referirse a lo real o imaginario tiene que tomar decisión de qué manera presentarlo: si a través del discurso "objetivo", en que no se nota la existencia de un enunciador individual, si utilizando el discurso "subjetivo", en el que "el enunciador se confiesa explícitamente o se reconoce implícitamente como la fuente evaluativa de la afirmación”.

Hasta este momento han surgido varias tipologías y clasificaciones del lenguaje evaluativo. (BRALCZYK, 1995, 2001; FUENTES RODRÍGUEZ; ALCAIDE LARA, 2002; KERBRAT-ORECCHIONI, 1986; MARTIN; WHITE, 2005; PUZYNINA, 1992) No obstante, en el presente trabajo seguimos la propuesta de la lingüista polaca Puzynina $(1984,1992,1997)$, ya 
que creemos que merece la pena sacar provecho de trabajos desarrollados por los investigadores que se ocupan de lenguas de menor alcance, a veces pertenecientes a otro sistema de conceptualización de la realidad, un sistema micro, que funciona dentro del sistema macro europeo. (WILK-RACIĘSKA, 2009, 2012) Seguimos a Puzynina (2014), que cree que el punto de partida para los estudios sobre lenguaje evaluativo se encuentra en la importancia de este tipo de lenguaje para la vida social del ser humano, ya que pensamos y nos comunicamos entre sí basándonos en lo que consideramos malo o bueno.

Así, Puzynina (1992) divide los mecanismos que marcan la evaluación en dos tipos: los elementos paralingüísticos y los elementos lingüísticos. En cuanto al primer tipo, en términos generales son elementos fónicos o entonación, de los que no nos ocupamos en el presente artículo. En cambio, para los elementos lingüísticos la autora propone la siguiente categorización: (1) elementos flexivos; (2) elementos sintácticos; (3) elementos de formación de palabras; (4) elementos léxicos; y (5) tropos estilísticos, tales como metáfora, comparaciones, ironía, símbolos, hipérbole y eufemismos. A pesar de que la tipología cubre muchos elementos, este trabajo se enfoca solamente en la cuestión de componentes léxicos. Consideramos que el estudio, por ejemplo, de tropos estilísticos requiere un análisis aparte, mucho más detallado.

Puzynina (1992) sostiene que el léxico es el recurso primordial de valoración y divide las palabras en dos tipos: las palabras que evalúan de forma primaria, y las que lo hacen de forma secundaria. Por lo que se refiere al primer grupo, son los vocablos que manifiestan la evaluación directamente, como sustantivos, adjetivos o adverbios. Algunos encierran elementos que indican la intensidad, por ejemplo, estupendo, horroroso. Además, pueden marcar valores que estrechamente se vinculan a la religión o la moralidad. En cambio, si se trata de la evaluación secundaria, se nota una fusión de elementos evaluativos y descriptivos. En este caso el factor evaluativo puede existir en la definición o manifestarse a través de un contexto dado. Asimismo, es imprescindible subrayar que hay palabras que despiertan connotaciones positivas o negativas dependiendo de la cultura en la que se aplican.

\section{Metodología}

Para el presente análisis del lenguaje evaluativo hemos escogido el lenguaje de las redes sociales, en concreto el de Instagram. Como ya hemos mencionado, debido a su desarrollo en los últimos años, consideramos la 
plataforma como un espacio muy bien preparado para publicar mensajes de varia índole. Por consiguiente, en lo que atañe al corpus, para el análisis nos han servido 78 posts publicados entre el 13 de marzo y el 12 de abril de 2020, es decir, a principios de la pandemia del covid-19. Los mensajes se han sacado de ocho perfiles de Instagram de carácter \#studygram seleccionados de forma manual, a base de los siguientes criterios:

- se han elegido de forma aleatoria;

- han aparecido en los resultados de búsqueda del hashtag \#studygramespaña;

- los resultados de búsqueda han aparecido en la bandeja de "destacados";

- sus mensajes, en general, son más elaborados y no contienen solamente los hashtags;

- tienen entre 1.000 y 2.000 seguidores.

En los mensajes del corpus se han seleccionado los ejemplos que tratan de tres cuestiones principales: (1) el covid-19 junto con el confinamiento y la cuarentena; (2) estado de ánimo; y (3) estudios. La búsqueda se ha hecho de manera manual, lo que nos ha permitido clasificar los vocablos tomando en consideración todo el contexto del mensaje y, por tanto, nos ha ayudado a evitar los posibles malentendidos que en algunos casos pudieran surgir. Asimismo, subrayamos que las tres categorías que hemos establecido no presentan límites claros y bien definidos, ya que un solo mensaje puede pertenecer a cada uno de estos grupos. La división que proponemos la tratamos más bien como una herramienta que nos ayuda a estructurar este trabajo.

La siguiente parte del estudio, es decir, el análisis de contenido ha tenido carácter cualitativo y se ha focalizado en analizar con más detalles el nivel léxico del corpus. Conviene destacar que el análisis de contenido, por lo general, no pretende analizar el estilo del texto, sino que enfoca su búsqueda en los vocablos u otros símbolos que modelan el contenido de los mensajes y configuran la comunicación interhumana. Como señala López Noguero (2002, p. 173), "esa técnica se constituye en un instrumento de respuesta a esa curiosidad natural del hombre por descubrir la estructura interna de la información, bien en su composición, en su forma de organización o estructura, bien en su dinámica". A nuestro modo de ver, tal análisis puede resultar una herramienta muy útil a la hora de estudiar el léxico evaluativo que ha sido analizado en el amplio contexto social del covid-19 y -en el caso de los estudiantes-, de la enseñanza online. 


\section{Resultados}

El análisis de los posts en las cuentas tipo \#studygram, tal y como ya hemos mencionado anteriormente, ha revelado que las publicaciones de los estudiantes se refieren, por un lado, directamente al covid-19, el confinamiento y la cuarentena y, por el otro, a los estados de ánimo o a las cuestiones relacionadas con estudio y preparaciones para las oposiciones, también en el contexto actual de la pandemia.

A continuación, presentaremos algunos de los ejemplos del lenguaje evaluativo divididos de acuerdo con los temas tratados por los usuarios de Instagram. Asimismo, nos gustaría subrayar que todos los ejemplos son anónimos, ya que -de acuerdo con las características de la plataforma Instagram- los mismos usuarios tienen derecho a cambiar sus nombres y, por tanto, puede que resulte imposible encontrarlos después de algún tiempo.

\subsection{Covid-19, confinamiento y cuarentena}

Para empezar, se analizan los fragmentos en las que los usuarios se refieren al principio de la situación, cuando el gobierno español llevó a cabo las primeras medidas, y a las personas que todavía no se daban cuenta de la gravedad del asunto:

(1) Aprovecho el post de hoy para hacer un llamamiento con respecto a la nueva situación que tenemos en España. Se han tomado medidas importantes y necesarias de cerrar colegios, universidades y demás, hagámoslo bien y quedémonos en casa, de nada sirve cerrar estos sitios si vamos a salir todos a la calle como si fueran vacaciones. Pensemos en los demás, no se trata solo de nosotros, seamos un poco solidarios \#yomequedoencasa [...]. (cuenta A, 13/03/2020)

(2) Otra cosa que me parece muy mal es que las personas que viven en Madrid se han venido a su casa de la playa, consecuencia, primer caso positivo en mi pueblo $⿴ 囗 \square$ creo que todavía no son conscientes de la gravedad del asunto o que les importa una mierda el resto del mundo [...]. (cuenta B, 13/03/2020)

(3) Toca quedarse en casa haciendo la cuarentena POR TODOS [...]. (cuenta C, 19/03/2020)

En lo que atañe a los ejemplos (1-3), podemos observar que la situación despierta muchas emociones entre la gente. Los usuarios aplican adjetivos que pertenecen al léxico que evalúa de forma primaria, como 
importante, necesario, consciente, la que destaca la actitud seria de los estudiantes hacia el problema. De la misma manera, el adjetivo bien, con valor axiológico transferido directamente, se relaciona con lo que nosotros, la gente común, podemos hacer en el caso de la enfermedad.

Otra cuestión, muy importante y frecuente en la época del covid-19, es el uso del verbo quedarse. Aunque a primera vista parece no transmitir ningún valor en el contexto de la pandemia que se extendió rápidamente, pero se ha convertido en un vocablo que evalúa de forma secundaria. Así, en la sociedad quedarse en casa y no salir sin necesidad se ha empezado a entender como sinónimo de un comportamiento solidario y responsable hacia otros y a sí mismo. Podemos observarlo en el ejemplo (2), en lo cual la autora se refiere a los viajes durante la pandemia. Utiliza el adverbio mal, calificando así de manera negativa el comportamiento de las personas que han venido a la playa. De la misma forma, la expresión final les importa una mierda el resto del mundo aumenta aún más las sensaciones negativas hacia las personas que viajan.

En cuanto al nivel argumentativo de los mensajes, el léxico aplicado en los ejemplos (1-3) permiten sacar la conclusión de que la pandemia es un problema muy grave y, por lo tanto, la gente debería hacer todo lo posible para ayudar a los médicos y ser más responsables respecto a otras personas.

Otro tema del que se habla en las cuentas \#studygram es el confinamiento, lo que muestran los ejemplos (4-6):

(4) El confinamiento es un asco pero me está ayudando mucho participar (junto a otras compis opositoras) en las sesiones de estudio conjuntas en Zoom, organizadas por @ tiempopapeles [...]. (cuenta D, 23/03/2020)

(5) Día X de la cuarentena (mejor no contarlos que nos ponemos nerviosos) [...]. (cuenta A, 17/03/2020)

(6) Día X de la cuarentena-confinamiento-pesadilla [...]. (cuenta A, $27 / 03 / 2020)$

En este caso notamos que las autoras hablan del confinamiento en términos de asco (4) o pesadilla (6). Son palabras que evalúan negativamente de forma primaria, que no dependen del contexto. Así los internautas entienden la situación como algo desagradable o una preocupación grave y continua. Asimismo, en el ejemplo (5) la autora utiliza el adjetivo nervioso con referencia a las personas que desde hace ya algún tiempo no salen de casa. Si tomamos 
en consideración los ejemplos (4) y (6), podemos entender las causas de este posible estado de ánimo de la gente.

La siguiente perspectiva de la pandemia del covid-19 muestra cómo los \#studygrammers hablan de los sanitarios y, en general, de los trabajadores de la sanidad pública:

(7) PD: mi opohermana que es enfermera se ha ido a trabajar al Hospital de Tomelloso. Un aplauso para todos los sanitarios que se están en primera línea de batalla dándolo todo por los demás, y con unas condiciones de seguridad pésimas [...]. (cuenta A, 27/03/2020)

(8) Hoy mi post va dedicado a mi hermana, a mi amiga y a todos los sanitarios, que están trabajando en primera línea contra este maldito virus, sin protección y sin nada [...]. (cuenta A, 31/03/2020)

En el ejemplo (7) la autora recurre al vocablo que pertenece al lenguaje evaluativo de forma primaria. El uso del sustantivo aplauso muestra claramente su actitud positiva hacia todo lo que hacen los sanitarios españoles. Tal opinión se ve aún más fuerte cuando observamos el léxico aplicado para las condiciones en las que se trabaja. Así, en el caso (7) se utiliza el adjetivo pésimo, el superlativo absoluto del adjetivo malo, lo que nos puede llevar a la conclusión de que la situación es de verdad muy grave. Asimismo, en el ejemplo (8) vemos que la autora se sirve de los sustantivos protección y nada. En cuanto al sustantivo nada, nos encontramos ante la evaluación primaria, mientras que si se trata de la palabra protección, aunque por su naturaleza puede pertenecer al léxico que evalúa de forma primaria, en el caso del covid-19 es fuertemente contextualizada y, por tanto, pertenece al lenguaje evaluativo secundario. El mensaje es marcado por el uso de la preposición sin que denota carencia o falta de algo. En este caso, el trabajo sin protección o, en general, sin nada merece aún más respeto.

El contexto del covid-19 en el que aparecen los mensajes nos permite concluir que la situación de la sanidad pública y, en particular, de los sanitarios, es muy seria. Por consiguiente, refiriéndose también a otros mensajes publicados por los estudiantes, deberíamos tomar el problema en serio y no arriesgar la vida de los demás. 


\subsection{Estado de ánimo}

La segunda categoría que hemos establecido para organizar el presente trabajo se refiere al estado de ánimo de los estudiantes, aunque los mensajes tratan también de otros asuntos.

Veamos los ejemplos donde los autores presentan la actitud positiva hacia la situación:

(9) Cuarto día de confinamiento. De momento lo llevo bien, porque cuando me agobio un poquito me voy a la terraza [...]. (cuenta $\mathrm{B}$, 16/03/2020)

(10) ¿Có mo lleváis estos días? Yo la verdad que estoy rindiendo bastante 울우. Espero que vosotros también! (cuenta E, 18/03/2020)

En el mensaje (9) notamos que el usuario habla de su estado de ánimo a principios del confinamiento y recurre al adverbio bien que pertenece al vocabulario que evalúa de forma primaria. De igual manera, la evaluación de forma primaria se utiliza también en el ejemplo (10), en el que el verbo rendir nos puede llevar a la conclusión de que la estudiante vence la situación del covid-19.

El siguiente ejemplo habla de estado de ánimo con vínculos a los estudios. Así podemos observar que los estudios, el tema principal de las cuentas \#studygram, se siguen explorando también en tiempos de pandemia:

(11) Hoy estoy contenta, porque ayer cumplí los objetivos del día (creo que es el primer día, desde que estamos en Estado de Alarma, que los cumplo . 20 (). Así que estoy motivada, y esta mañana también los estoy cumpliendo

Como en el caso de los ejemplos anteriores, también en el mensaje (11) podemos observar que la evaluación se realiza de forma primaria. Los adjetivos contenta y motivada transmiten directamente la información que la autora está de buen humor.

Por si se trata a la conclusión a la que podemos llegar a base de los mensajes (9-11), conviene resaltar que tal uso de léxico evaluativo puede influir en el posible aumento de la motivación de los seguidores de las cuentas \#studygram, lo que se relaciona también con la finalidad general de cuentas de este tipo.

En cuanto a la actitud negativa en tiempos de pandemia, aunque nuestro análisis no es cuantitativo, se nota que los casos en los que los 
estudiantes escriben que se sienten agobiados o preocupados son más comunes. Veamos algunos ejemplos:

(12) Estos días están siendo un poco raros... el otro día tuve un momento de bajón total, la incertidumbre que rodea a todo el tema de las opos fue lo que hizo que me viniera abajo [...]. (cuenta E, 28/03/2020)

En el ejemplo (12) se habla del estado de ánimo, pero en el contexto de las oposiciones a las que se prepara la autora. Notamos el uso del sustantivo bajón que evalúa de forma secundaria, a base de su definición, ya que se refiere a "deterioro acusado de la salud o el ánimo" (DRAE, 2013). Además, el mensaje se refuerza con el adjetivo total, lo que puede subrayar que el día de la \#studygrammer de verdad fue muy deprimido.

En el post aparece también el sustantivo incertidumbre cuya valoración se halla en el factor descriptivo de falta de certeza y, por consiguiente, pertenece al lenguaje evaluativo de forma secundaria. La locución verbal venirse abajo, que se entiende como "derrumbarse", presenta un matiz metafórico a través del que hablamos del estado de ánimo en términos de movimiento hacia abajo o arriba. (LAKOFF; JOHNSON, 1980) Por tanto, consideramos que es un ejemplo de la evaluación de forma secundaria.

(13) [...] Día X del confinamiento. Las noticias no son nada alentadoras, la verdad que cada vez que las veo me da mucha ansiedad, miedo, preocupación... [...] (cuenta A, 27/03/2020)

Las emociones negativas que se mencionan en el ejemplo (13), es decir, ansiedad, miedo y preocupación, pertenecen al léxico evaluativo de forma secundaria, cuyo factor axiológico se indica a través de la descripción y/o definición de la palabra. La autora se sirve también del adjetivo alentadoras, pero lo utiliza junto con la negación no y el adverbio indefinido nada, lo que de modo descriptivo cambia totalmente la perspectiva desde lo positivo hacia lo negativo.

(14) Hola a todos ¿ ¿Cómo estáis llevando la cuarentena? A mi me agobia mucho esto de tener que estar en casa por obligación, pero es lo mejor para todos, así que a aprovechar el tiempo lo mejor que se pueda [...]. (cuenta C, 21/03/2020)

El léxico que evalúa de forma secundaria se puede observar también en el ejemplo (14), en el que la autora utiliza el verbo agobiar para explicar cómo se siente quedándose en casa por obligación. Como en los casos anteriores, 
el matiz evaluativo se percibe en la descripción de este tipo de sentimientos, ya que la definición lo explica como "imponer a alguien actividad o esfuerzo excesivos, preocupar gravemente, causar gran sufrimiento", pero también "rendir, deprimir o abatir" (DRAE, 2013).

(15) La concentración va y viene por momentos, descanso mal de noche, tengo picos de ansiedad y los brotes de fibromialgia van y vienen a su antojo [...]. (cuenta D, 9/04/2020)

En el ejemplo (15) podemos notar las referencias a problemas de salud, como ansiedad o fibromialgia, que, por su naturaleza de ser enfermedad, se entienden de manera negativa. Aunque los valores transmitidos por estas palabras son muy bien perceptibles, los consideramos ejemplos del lenguaje evaluativo de forma secundaria, ya que, por un lado, tienen carácter descriptivo y, por otro, su lectura en el contexto de la pandemia puede referirse a algunas connotaciones más fuertes de lo normal.

Asimismo, el autor utiliza el adverbio mal que pertenece al grupo de palabras que evalúan de forma primaria. De esta manera, su aplicación con referencia al descanso nos puede llevar a la conclusión de que toda la situación también afecta a los estudiantes, aunque no sufren directamente a causa del covid-19.

(16) Llevo todo el día en casa, solamente he salido a sacar la basura, esto del coronavirus me está dando ya un poco de yuyu C, $12 / 03 / 2020)$

En el ejemplo (16) se nota el uso de la expresión coloquial dar yuyu que transmite los valores de forma secundaria. Aunque no aparece en el Diccionario RAE, yuy se explica como "una clase de miedo muy particular, aquel que es producido por algo paranormal o esotérico". (LÓPEZ, 2019) Así, se entiende que el covid-19 da miedo a todos, incluso a los estudiantes.

Los mensajes (12-16), aunque tienen carácter negativo y aluden a los problemas muy serios para los estudiantes, nos permiten sacar algunas conclusiones más generales que están estrechamente relacionadas con el propio carácter de las cuentas \#studygram, es decir, presentan que el problema de la pandemia afecta a todos y que cada uno tiene que aprender a organizarse en esta situación. 


\subsection{Estudios}

Con respecto al tema de estudios, hemos seleccionado solamente los que se relacionan con el problema del covid-19, aunque conviene resaltar que, por la naturaleza de las cuentas analizadas, la cuestión de estudios y aprendizaje se menciona en todos los posts.

(17) En esto se está basando mi cuarentena. La verdad que mi concentración para estudiar es nula, así que aprovecho para leer y adelantar libros que tenía abandonados... [...]. (cuenta F, 28/03/2020)

(18) Ayer el día, en lo que a estudio respecta, no fue nada bien. Concentración nula, mucho ruido, una obra que no paraba, ni los tapones servían... (cuenta A, 17/03/2020)

(19) Las oposiciones de educación infantil se aplazan al $2022=$. He de confesar que me ha desmotivado mucho en cuanto al estudio pero seguiré con ello, poco a poco 5 . (cuenta E, 4/04/2020)

(20) Por medio se ha juntado el brote de Coronavirus, y nos piden que nos quedemos en casa por lo que, lo último que me apetece ahora con tantas preocupaciones, es ponerme a estudiar. Retomaré a partir del lunes. (cuenta D, 14/03/2020)

Observamos que en los ejemplos (17-19) el lenguaje evaluativo se utiliza para referirse a la concentración y motivación. Así, en los mensajes (17-18) los autores aluden a la concentración utilizando el adjetivo nulo que significa, entre las acepciones, "falto de valor y fuerza para obligar o tener efecto, por ser contrario a las leyes, o por carecer de las solemnidades que se requieren en la sustancia o en el modo" (DRAE, 2013). A base del carácter descriptivo de la palabra y de su definición consideramos que es un ejemplo del lenguaje evaluativo de forma secundaria. Asimismo, en el ejemplo (18) aparece el adverbio bien cuyo significado se actualiza a través del adverbio indefinido nada y la negación. Igual como en el caso (13), podemos notar el cambio de la perspectiva desde lo positivo hacia lo negativo.

La cuestión de motivación se menciona en el ejemplo (19) cuando el autor se aprovecha del verbo desmotivar para presentar su actitud hacia decisiones tomadas respecto al cambio de la fecha de las oposiciones. El verbo lo entendemos, por un lado, a base de su definición y, por otro, tomando en consideración el contexto en el que se utiliza. De la misma manera se entiende el adjetivo último utilizado por el estudiante en el ejemplo 
(20). Observamos que transmite los valores negativos de forma secundaria por medio del contexto de falta de motivación en tiempos de pandemia.

(21) A por otras dos semanas más de cuarentena Un día más es un día menos, así que empecemos la semana con positividad, sacando el lado positivo de todo esto (aunque sea difícil) y aprovechando las semanas. Yo \#mequedoencasa [...]. (cuenta F, 6/04/2020)

(22) Vamos al lío! Comenzamos semana, espero que sea productiva y que tengamos buenas noticias con respecto al covid-19. (cuenta A, $6 / 04 / 2020)$

A diferencia de los ejemplos (17-20), en los casos (21-22) observamos el lenguaje evaluativo que transmite posturas positivas de los estudiantes. Tanto el sustantivo positividad como los adjetivos positivo y bueno evalúan la situación de forma primaria. Si se trata del adjetivo productiva que aparece en el mensaje (22), creemos que su valor positivo se transmite de forma secundaria. Además, la lectura de todo el ejemplo muestra que el uso del léxico evaluativo que se refiere a lo positivo tiene por objetivo motivar o animar a los seguidores, tal vez otros estudiantes, que aprovechen la semana para estudiar.

(23) Vamos a ver si somos capaces de acostumbrarnos a estudiar en casa aquellos que antes no podíamos. Es un nuevo reto. (cuenta $A$, $15 / 03 / 2020)$

El último de los ejemplos alude a la situación sin precedentes, algo que hasta cierto momento ninguno no ha vivido. En este contexto el sustantivo reto utilizado por el usuario se entiende de manera positiva y pertenece al léxico que evalúa de forma secundaria. Además, el adjetivo nuevo, propiamente evaluativo de forma primaria, aún refuerza el punto de vista del mensaje.

\section{Consideraciones finales}

El estudio cualitativo del lenguaje evaluativo utilizado por los estudiantes en las cuentas del tipo \#studygram en Instagram ha permitido observar cómo el problema de la pandemia del covid-19 se conceptualiza entre los jóvenes.

En primer lugar, hemos observado que los usuarios de Instagram se sirven tanto del léxico propiamente evaluativo, que transmite valores de 
forma primaria, como del vocabulario que está fuertemente arraigado en el contexto. Así, en las cuentas \#studygram hemos analizado los adjetivos, como bueno, malo o positivo, que pertenecen al primer grupo, pero también los términos como yuyu, ansiedad, fibromialgia o quedarse en casa, que requieren un conocimiento extralingüístico más amplio.

En segundo lugar, hemos verificado que el nivel argumentativo de los mensajes publicados en este tipo de cuentas está presente dentro de la propia lengua de los usuarios, por un lado, y depende del carácter y de las funcionalidades de la plataforma Instagram, por otro. Así, el léxico axiológico que evalúa de manera positiva se puede utilizar con el objetivo de animar a otros a que se comporten de alguna manera, por ejemplo a que no se rindan y sigan estudiando.

Nuestros resultados sugieren que el mundo virtual de las redes sociales, y en este caso de Instagram, ya está estrechamente relacionado con la vida de buena parte de los estudiantes que lo tratan como si fuera una posibilidad más de contactarse con la gente. No obstante, somos conscientes de que el fenómeno de las cuentas \#studygram necesitaría un estudio mucho más profundo, no solamente desde la perspectiva lingüística, sino también en el ámbito de las ciencias sociales o de comunicación.

En suma, creemos que el presente trabajo aporta información valiosa para entender el lenguaje de los jóvenes utilizado en las redes sociales. Asimismo, nos parece oportuno resaltar que en este análisis se ha estudiado solamente uno de los mecanismos argumentativos aplicados en las cuentas \#studygram. El discurso lo podemos investigar también desde otros puntos de vista. Así un estudio más sistemático y profundo podría examinar otras maneras de transmisión de valores, las cuestiones de operadores y conectores argumentativos o los tropos estilísticos, como, por ejemplo, la metáfora o la ironía.

\section{Referencias}

AGOBIAR. In: DICCIONARIO da Real Academia Española. Madrid: RAE, 2013. Disponible en: https:// dle.rae.es/agobiar. Acceso en: 4 oct. 2021.

ÁLVAREZ-ROSA, C. V.; ROMERO LÓPEZ, Á. M. La prensa digital en tiempos de la Covid-19: análisis lingüístico de los titulares publicados en Ávilared. Revista de Comunicación y Salud, Madrid, v. 10, n. 2, p. 385-396, 2020. DOI: https://doi. org/10.35669/rcys.2020.10(2).385-396. 
ALVES, R.; LOPES, T.; PRECIOSO, J. Teachers' well-being in times of Covid-19 pandemic: factors that explain professional well-being. IJERI: International Journal of Educational Research and Innovation, Sevilla, n. 15, p. 203-217, 2020. DOI: https:/ / doi.org/10.46661/ijeri.5120.

ANSCOMBRE, J.-C.; DUCROT, O. La argumentación en la lengua. Madrid: Gredos, 1994.

ARAB, L. E.; DÍAZ, G. A. Impacto de las redes sociales e internet en la adolescencia: aspectos positivos y negativos. Revista Médica Clínica Las Condes, Las Condes, v. 26, n. 1, p. 7-13, 2015. DOI: https://doi.org/10.1016/j. rmclc.2014.12.001.

ARGIÑANO, J.-L.; GOIKOETXEA BILBAO, U. Análisis de los titulares y las fotografías de portada en España en el contexto de la crisis del coronavirus: protagonistas, frames y lenguaje bélico. Revista de Comunicación y Salud, Madrid, v. 10, n. 2, p. 1-23, 2020. DOI: https://doi.org/10.35669/rcys.2020.10(2).1-23.

BAJÓN. In: DICCIONARIO da Real Academia Española. Madrid: RAE, 2013. Disponible en: https://dle.rae.es/bajon. Acceso en: 4 oct. 2021.

BASTARD, P. et al. Autoantibodies against type I IFNs in patients with lifethreatening COVID-19. Science, Washington DC, v. 370, n. 6515, p. eabd4585, 2020. DOI: https://doi.org/10.1126/science.abd4585.

BERNAL GALINDO, R. M. Herramientas telemáticas para la comunicación educativa: catalogación, análisis y posibilidades de uso de los blogs. 2015. Tesis (Doctoral en Pedagogía) - Universidad de Murcia, Murcia, 2015.

BONGARRÁ, A. C. Docentes de la "Generación Y". Reflexión Académica en Diseño y Comunicación, Buenos Aires, v. 12, n. 15, p. 169-173, 2011.

BRALCZYK, J. Ograniczony świat propagandy. In: ROKOSZOWA J. (Ed.). Nowomowa. Materiały z sesji nauko-wej poświęconej problemom współczesnego języka polskiego odbytej na Uniwersytecie Jagiellońskim w dniach 16 i 17 stycznia 1981. Londyn: Polonia, 1995. p. 105-132.

BRALCZYK, J. O jesyku polskiejpropagandy politycznej lat siedemdziesiatych. Warszawa: Trio, 2001. v. 1.

BUCZEK, P. Instagram jako miejsce komunikacji wizualnej mediów z odbiorcami. Naukowy Przeglad Driennikarski, [s. l.], v. 24, n. 4, p. 131-141, 2017.

CABRER BORRAS, B.; RICO, P. Impacto económico del sector turístico en España. Studies of Applied Economics, Almería, v. 39, n. 1, 2020. DOI: https://doi. org/10.25115/eea.v39i1.3599. 
CLAVIJO NAULA, M.; CABRERA, S. Análisis del discurso científico en Twitter durante el primer trimestre de la pandemia de Covid-19 en Ecuador. \#PerDebate, Quito, v. 4, n. 1, p. 128-155, 2020. DOI: https://doi.org/10.18272/pd.v4i1.1882. CONDE, M. A.; FORTEZA MARTÍNEZ, A.; ANDRADE MARTÍNEZ, C. M. Análisis de la capacidad de liderazgo y el carisma de los principales youtubers españoles a través del diseño de un modelo de estudio de la competencia mediática en YouTube. 3C TIC: Cuadernos de Desarrollo Aplicados a las TIC, Logroño, v. 9 , n. 3, p. 17-41, 2020.

CONRAD, S.; BIBER, D. Adverbial marking of stance in speech and writing. In: HUNSTON, S.; THOMPSON, G. (Ed.). Evaluation in text. Authorial stance and the construction of discourse. New York: Oxford University Press, 2020. p. 56-73.

DANTAS, L. F. S.; DECCACHE-MAIA, E. Divulgação científica no combate às fake news em tempos de Covid-19. Research, Society and Development, [s. l.], v. 9, n. 7, p. e797974776, 2020. DOI: https://doi.org/10.33448/rsd-v9i7.4776.

DÍEZ GUTIÉRREZ, E. J.; GAJARDO ESPINOZA, K. Políticas educativas en tiempos de coronavirus: la confrontación ideológica en España. Revista Internacional de Educación para la Justicia Social, Madrid, v. 9, n. 3, p. 83-101, 2020. DOI: https:// doi.org/10.15366/riejs2020.9.3.005.

DOMINGUES ALMEIDA, A. A.; SANTOS, E. S. Ciência, opinião e fake news em tempos de coronavírus: Conceptualizações em memes sob a abordagem da linguística cognitiva. Pensares em Revista, São Gonçalo, v. 19, p. 56-76, 2020. DOI: https://doi.org/10.12957/pr.2020.52642.

ESCANDELL VIDAL, M. V. Introducción a la pragmática. Barcelona: Ariel, 2007. ESPINOZA, E.; TINOCO IZQUIERDO, W. E.; SÁNCHEZ BARRETO, X. R. Características del docente del siglo XXI. Olimpia, Bayamo, v. 14, n. 4, p. 39-53, 2017.

FERNÁNDEZ MARTÍNEZ, M. M. et al. La tecnología en el ámbito educativo ante el Covid: una apuesta por los MOOC como estrategia formativa en el contexto universitario. IJERI: International Journal of Educational Research and Innovation, Sevilla, v. 15, p. 130-142, 2020. DOI: https://doi.org/10.46661/ijeri.5133.

FONT MARSAL, M. Comunicar la moda: de las revistas femeninas al fenómeno Instagram. Barcelona: Universidad Autónoma de Barcelona, 2015.

FUENTES RODRÍGUEZ, C.; ALCAIDE LARA, E. R. Mecanismos lingüisticos de la persuasión: cómo convencer con palabras. Madrid: Arco/Libros, 2002.

FUENTES RODRÍGUEZ, C.; ALCAIDE, E. R. La argumentación lingüistica y sus medios de expresión. Madrid: Arco/Libros, 2007. 
GARCÍA MARÍN, D. Infodemia global. Desórdenes informativos, narrativas fake y fact-checking en la crisis de la Covid-19. Elprofesional de la información, León, v. 29, n. 4, p. e290411, 2020. DOI: https://doi.org/10.3145/epi.2020.jul.11.

GARCÍA MEJÍA, R. O.; GARCÍA VERA, C. E. Metodología STEAM y su uso en matemáticas para estudiantes de bachillerato en tiempos de pandemia Covid-19. Dominio de las Ciencias, Manta, v. 6, n. 2, p. 163-180, 2020.

GONZÁLEZ HERNANDO, C.; VALDIVIESO LEÓN, L.; VELASCO GONZÁLEZ, V. Estudiantes universitarios descubren redes sociales y edublog como medio de aprendizaje. RIED. Revista Iberoamericana de Educación a Distancia, Madrid, v. 23, n. 1, p. 223-239, 2020. DOI: https://doi.org/10.5944/ ried.23.1.24213.

GUTIÉRREZ COBA, L.; COBA GUTIÉRREZ, P.; GÓMEZ DIAZ, J. A. Las noticias falsas y desinformación sobre el Covid-19: análisis comparativo de seis países iberoamericanos. Revista Latina de Comunicación Social, Madrid, n. 78, p. 237264, 2020. DOI: https:// doi.org/10.4185/RLCS-2020-1476.

HABERMAS, J. Strukturalne przeobrażenia sfery publicznej. Warszawa: Wydawnictwo Naukowe PWN, 2008.

HARKER, M. ¿Qué es el \#studygram y cómo sacarle el mejor provecho? Las lecturas de Mina, [s. l.], 24 ene. 2019. Disponible en: https://bit.ly/3m6BiOx. Acceso en: 10 nov. 2020.

HUNSTON, S.; SINCLAR, J. A local grammar of evaluation. In: HUNSTON, S.; THOMPSON, G. (Ed.). Evaluation in text. Authorial stance and the construction of discourse. New York: Oxford University Press, 2000. p. 74-101.

HUNSTON, S.; THOMPSON, G. Evaluation in text. Authorial stance and the construction of discourse. New York: Oxford University Press, 2000.

IZQUIERDO IRANZO, P.; GALLARDO ECHENIQUE, E. E. Estudigramers: influencers del aprendizaje. Comunicar, Andalucía, v. 28, n. 62, p. 115-125, 2020. DOI: https://doi.org/10.3916/C62-2020-10.

KASHYAP, A.; RAGHUVANSHI, J. A preliminary study on exploring the critical success factors for developing COVID-19 preventive strategy with an economy centric approach. Management Research, Bingley, v. 18, n. 4, p. 357-377, 2020. DOI: https://doi.org/10.1108/MRJIAM-06-2020-1046.

KERBRAT-ORECCHIONI, C. La enunciación de la subjetividad en el lenguaje. Buenos Aires: Hachette, 1986.

KOPEĆ, J. Hashtagi, ćwierkanie i świat jako baza danych. Antropologia surfingu, [s. l.], 5 feb. 2015. Disponible en: https://bit.ly/3ofG52N. Acceso en: 19 nov. 2020. 
LAKOFF, G.; JOHNSON, M. Metáforas de la vida cotidiana. Madrid: Cátedra, 1980. LIU, Y. Social media tools as a learning resource. Journal of Educational Technology Development and Exchange, Prescott Valey, v. 3, n. 1, 2010. DOI: https://doi. org/10.18785/jetde.0301.08.

LLANO GUIBARRA, N. I.; AGUILA SÁNCHEZ, J. C. Conferencias de prensa y Covid-19: explorando la respuesta gubernamental mexicana desde la comunicación en salud. Revista Española de Comunicación en Salud, Madrid, v. 11, n. 1, p. 128-141, 2020. DOI: https://doi.org/10.20318/recs.2020.5451.

LLORENTE HERAS, R. Impacto del Covid-19 en el mercado de trabajo; un análisis de los colectivos vulnerables. Documentos de Trabajo, Alcalá de Henares, v. 2, p. 1-29, 2020.

LLORENTE, A. Mejores hashtags para Instagram en 2020. Printful, [s. l.], 9 feb. 2019. Disponible en: https://bit.ly/3CRyLyw. Acceso en: 28 oct. 2020.

LO CASCIO, V. Gramática de la argumentación: estrategias y estructuras. Madrid: Alianza, 1998.

LÓPEZ NOGUERO, F. El análisis de contenido como método de investigación. Educación XXI, Madrid, v. 4, p. 167-180, 2002.

LÓPEZ, A. Dar yuyu. Ya está el listo que todo lo sabe, Barcelona, abr. 2019. Disponible en: https://bit.ly/39NkVk6. Acceso en: 9 dic. 2020.

MARTÍN ZORRAQUINO, M. A.; PORTOLÉS, J. Los marcadores del discurso. In: BOSQUE, I.; DEMONTE, V. (Ed.). Gramática descriptiva de la lengua española. Madrid: EspasaCalpe, 1999. p. 4051-4213.

MARTIN, J. R.; WHITE, P. R. R. The language of evaluation: appraisal in English. New York: Palgrave Macmillan, 2005.

MARTIN, J. R; ROSE, D. Genre relations: mapping culture. London: Equinox Pub, 2008.

MEDINA, F. Instagram como recurso didáctico para desarrollar la escritura creativa: caso microrrelato. Cuaderno de Pedagogia Universitaria, Santiago de los Caballeros, v. 17, n. 33, p. 84-93, 2020. DOI: https://doi.org/10.29197/cpu. v17i33.380.

MEGÍA, C. Egoempollones, lo último en internet para presumir de apuntes molones. El Pais, Madrid, 18 mayo 2017. S Moda. Disponible en: https://bit. ly/3CYF0AL. Acceso en: 28 sept. 2021. 
MEHTA, P. et al. COVID-19: consider cytokine storm syndromes and immunosuppression. The Lancet, London, v. 395, n. 10229, p. 1033-1034, 2020. DOI: https://doi.org/10.1016/S0140-6736(20)30628-0.

MOEN, P.; PEDTKE, J. H.; FLOOD, S. Disparate disruptions: intersectional COVID-19 employment effects by age, gender, education, and race/ethnicity. Work, Aging and Retirement, Oxford, v. 6, n. 4, p. 207-228, 2020. DOI: https://doi. org/10.1093/workar/waaa013.

MOLINA AVENTOSA, P.; VALENCIANO VALCÁRCEL, J.; VALENCIA PERIS, A. Los blogs como entornos virtuales de enseñanza y aprendizaje en Educación Superior. Revista Complutense de Educación, Madrid, v. 26, p. 15-31, 2015. DOI: https://doi.org/10.5209/rev_RCED.2015.v26.43791.

MONTANER VILLALBA, S. La competencia en producción escrita en lengua inglesa mediante el blogging en el enfoque AICLE en la enseñanza secundaria obligatoria. El estudio del caso. In: DIOS MARTÍNEZ AGUDO, J.; MAYA RETAMAR, M. G.; GONZÁLEZ R. (Ed.). Educación bilingüe a debate. Práctica, investigación y formación del profesorado. Cáceres: Universidad de Extremadura, 2019. p. 79-95.

MONTEIRO, R. P. et al. Vício no Insta: propriedades psicométricas da escala Bergen de adição ao Instagram. Avances en Psicología Latinoamericana, Bogotá, v. 38, n. 3, p. 1-12, 2020. DOI: https://doi.org/10.12804/revistas.urosario.edu.co/ apl/a.8132.

MONTENOVO, L. et al. Determinants of disparities in COVID-19 job losses. NBER Working Paper Series, Cambridge, MA, 2020. DOI: https://doi.org/10.3386/ w27132.

MORALES CHÁVEZ, M. C. Coronavirus y discapacidad. Una población muy vulnerable. Acta Odontológica Venezolana, Caracas, v. 58, n. 1, p. 9-10, 2020.

MORENO MARTÍNEZ, N. M.; MORALES CEVALLOS, M. B. Covid-19 desde una óptica tecno-educativa a través de markerspaces. IJERI: International Journal of Educational Research and Innovation, Sevilla, v. 15, p. 57-72, 2020. DOI: https://doi.org/10.46661/ijeri.4898.

NAVARRO COLORADO, B. Aspectos retórico-comunicativos del desarrollo de los sitios web. In: BIENNIAL CONFERENCE, 14., 2003, Madrid. Anais [...]. Madrid: International Society of the History of Rhetoric, 2003. Disponible en: https://bit.ly/3odnQej. Acceso en: 19 nov. 2020.

NISHIURA, H. et al. Closed environments facilitate secondary transmission of coronavirus disease 2019 (COVID-19). Epidemiology, New Haven, 2020. DOI: https://doi.org/10.1101/2020.02.28.20029272 
NULO. In: DICCIONARIO da Real Academia Española. Madrid: RAE, 2013. Disponible en: https:/ / dle.rae.es/nulo. Acceso en: 4 oct. 2021.

OLMO, L. Studygram, la tendencia en redes sociales que convierte los apuntes en obras de arte. TICbeat, 19 mayo 2017. Disponible en: http://bityl.pl/jil8M. Acceso en: 6 de oct. 2021.

ORTEGA, I.; SOTO, I. Las universidades y la Generación Z. Universidad, sí, Madrid, 20 jun. 2016. Disponible en: https://bit.ly/3ifHzqd. Acceso en: 5 dic. 2020.

PASCUAL, M. Corrientes teóricas y aportes de estudios del lenguaje evaluativo al análisis del discurso. In: CONGRESO INTERNACIONAL DE LA ALED, 9., 2011, Belo Horizonte. Anais [...]. Belo Horizonte: Aled, 2011.

PERELMAN, C.; OLBRECHTS-TYTECA, L. Tratado de la argumentación: la nueva retórica. Madrid: Gredos, 1989.

PÉREZ-CURIEL, C.; VELASCO MOLPECERES, A. M. Impacto del discurso político en la difusión de bulos sobre Covid-19. Influencia de la desinformación en públicos y medios. Revista Latina de Comunicación Social, Madrid, n. 78, p. 65-97, 2020. DOI: https://doi.org/10.4185/RLCS-2020-1469.

PIRES FRANCO, H. L. et al. Fake news: mecanismos linguísticos de validação em destaque. Revista Transformar, Itaperuna, v. 14, p. 231-243, 2020.

PLANTIN, C. La argumentación. Barcelona: Ariel, 2005.

PUZYNINA, J. Je $\square$ zyk wartości. Warszawa: Wydawnictwo Naukowe PWN, 1992.

PUZYNINA, J. Kłopoty z nazwami wartości (i wartościami). Etnolingwistyka. Problemy Jezyka i Kultury, Lublin, v. 26, p. 7-20, 2014. DOI: https://doi. org/10.17951/et.2014.26.7.

PUZYNINA, J. O dyskursie oceniającym i dyrektywnym w tekstach prasy codziennej. Poradnik Jesykonyy, Warszawa, v. 2, p. 69-78, 1984.

PUZYNINA, J. Stowo-wartość-kultura. Lublin: Towarzystwo Naukowe Katolickiego Uniwersytetu Lubelskiego, 1997.

SÁNCHEZ PACHECO, C. L. Las redes sociales y las habilidades de enseñanza aprendizaje del siglo XXI. Eduser, Trujillo, v. 7, n. 1, p. 49-55, 2020.

SPURK, D.; STRAUB, C. Flexible employment relationships and careers in times of the COVID-19 pandemic. Journal of Vocational Behavior, Amsterdam, v. 119, p. 1034335, 2020. DOI: https://doi.org/10.1016/j.jvb.2020.103435. 
THE SOCIAL MEDIA FAMILY. VI Estudio sobre los usuarios de Facebook, Twitter e Instagram en España. Madrid: The Social Media Family, 2020. Disponible en: https:/ / bit.ly/3kOkpZF. Acceso en: 15 nov. 2020.

TIFFANY, K. Welcome to Studyblr: a beautiful, stressful wonderland. The Verge, New York, 9 mayo 2017. Disponible en: https://bit.ly/3kQDdrg. Acesso en: 12 nov. 2020.

TORRES, R.; FERNÁNDEZ, M. J. La política económica española y el Covid-19. Cuadernos de Información Económica, Madrid, v. 275, p. 1-7, 2020.

VERDÚ, D. La generación Z cambiará el mundo. El País, Madrid, 3 mayo 2015. Disponible en: https://bit.ly/3CRHDnO. Acceso en: 28 sept. 2021.

VILLA GRACIA, A. D.; CERDÁN MARTÍNEZ, V. Bulos durante la pandemia del Covid-19 en España: un estudio a través de Google Trends. Revista Latina de Comunicación Social, Madrid, n. 78, p. 169-182, 2020. DOI: https://doi.org/10.4185/ RLCS-2020-1473.

VILLASEÑOR LÓPEZ, K. et al. Cambios en el estilo de vida y nutrición durante el confinamiento por SARS-CoV-2 (Covid-19) en México: un estudio observacional. Revista Española de Nutrición Humana y Dietética, Pamplona, v. 25, n. 2, p. e1099, 2021.

VIVANCO SARAGURO, A. Teleducación en tiempos de Covid-19: brechas de desigualdad. CienciAmérica, [s. l.], v. 9, n. 2, p. 166-175, 2020. DOI: https://doi. org/10.33210/ca.v9i2.307.

VIZCAÍNO VERDÚ, A.; CONTRERAS PULIDO, P. ¡Soy músico youtuber! Construcción de la identidad artística a través del cover y la ficción. El caso de las violinistas Taylor Davis y Lindsey Stirling. Revista Mediterránea de Comunicación, [s. l.], v. 11, n. 1, p. 25-36, 2020. DOI: https://doi.org/10.14198/MEDCOM2020.11.1.1. WILK-RACIĘSKA, J. Entre la visión del mundo temporaly la aspectual: casos del español sudamericano. Katowice: Wydawnictwo Uniwersytetu Śląskiego, 2012.

WILK-RACIĘSKA, J. Od wizji śniata do opisu jezyykoznawczego w kategoriach lingwistyki kulturowej: Uwagi na temat hiszpańskiej syntagmy nominalnej. Katowice: Wydawnictwo Uniwersytetu Śląskiego, 2009.

Data de submissão: 11/12/2020. Data de aprovação: 15/07/2021. 